\title{
Factors Associated with Disruptive Behavioral Symptoms in Idiopathic Rapid Eye Movement Sleep Behavior Disorder
}

\author{
Soonkee Ryu, MD¹, Min Ji Kim, MD¹, Hayun Choi, MD², Hyuk Joo Lee, MD¹, In-Young Yoon, MD, PhD ${ }^{1,3}$ \\ 'Department of Neuropsychiatry, Seoul National University Bundang Hospital, Seongnam, Korea \\ 2Department of Psychiatry, Veteran Health Service Medical Center, Seoul, Korea \\ ${ }^{3}$ Department of Psychiatry, Seoul National University College of Medicine, Seoul, Korea
}

Received: June 1, 2018

Revised: June 15, 2018

Accepted: June 17, 2018

Correspondence

In-Young Yoon, $\mathrm{MD}, \mathrm{PhD}$

Department of Neuropsychiatry,

Seoul National University Bundang Hospital,

82 Gumi-ro 173beon-gil, Bundang-gu,

Seongnam 13620, Korea

Tel +82-31-787-7433

Fax +82-31-787-4058

E-mail iyoon@snu.ac.kr

ORCID

Soonkee Ryu

https://orcid.org/0000-0002-7087-1325 Min Ji Kim

https://orcid.org/0000-0002-6153-8811

Hayun Choi

https://orcid.org/0000-0002-8404-8006

Hyuk Joo Lee

https://orcid.org/0000-0002-3569-9603

In-Young Yoon

https://orcid.org/0000-0002-3995-8238

\begin{abstract}
Background and Objective Little is known about the factors associated with disruptive behaviors in idiopathic rapid eye movement (REM) sleep behavior disorder (iRBD). Thus, we report the demographic and clinical characteristics of $i R B D$ patients with disruptive behaviors.

Methods Patients diagnosed as having iRBD through video-polysomnography were included in the present study. The REM sleep behavior disorder questionnaire-Hong Kong (RBDQ-HK) was used to divide participants into two groups according to the presence or absence of disruptive behaviors. Neuropsychological tests as well as the Pittsburgh Sleep Quality Index (PSQI), Epworth Sleepiness Scale (ESS), and Beck Depression Inventory (BDI) were also conducted.

Results Of 188 iRBD patients, 160 patients showed more disruptive behaviors and were of a younger age ( $66.8 \pm 7.4$ vs. $70.5 \pm 6.0, \mathrm{p}=0.012)$, of a higher proportion of men $(69.4 \%$ vs. $39.3 \%, \mathrm{p}=$ $0.004)$, and showed lower Apnea-Hypopnea Indexes (AHI) $(9.2 \pm 0.9$ vs. $17.8 \pm 2.2, \mathrm{p}<0.001)$ than those without disruptive behavioral symptoms. No statistically significant differences in PSQI, ESS, $\mathrm{BDI}$, or neuropsychological testing were found. In multiple logistic regression analysis, age $\geq 70$ [odds ratio $(\mathrm{OR})=0.36,95 \%$ confidence interval $(\mathrm{CI})=0.15-0.87, \mathrm{p}=0.023$ ], male sex $(\mathrm{OR}=2.80$, $95 \% \mathrm{CI}=1.14-6.91, \mathrm{p}=0.025$ ), and score of item 4 (violent or aggressive dreams) of RBDQ-HK $(\mathrm{OR}=1.57,95 \% \mathrm{CI}=1.17-2.12, \mathrm{p}=0.003)$ all showed a significant association with disruptive behaviors.

Conclusions Age $<70$, male sex, lower AHI, and frequent violent or aggressive dreams are associated with an increased risk of disruptive behavioral symptoms in iRBD. Therapeutic plans including clonazepam medication should be individualized considering patients' demographic and clinical features.

Sleep Med Res 2018;9(1):46-52
\end{abstract}

Key Words Disruptive behaviors, REM sleep behavior disorder, RBDQ-HK.

\section{INTRODUCTION}

Rapid eye movement (REM) sleep behavior disorder (RBD) is a disorder characterized by the loss of atonia during REM sleep as well as dream enacting behavior [1,2]. The prevalence of RBD in the general population is approximately $0.5 \%$, and higher in the elderly population $[3,4]$. In today's aging society, research on RBD is of clinical significance as it is not only a parasomnia, but also recognized as a prodromal stage of neurodegenerative disorders. Up to 81$90 \%$ of people with idiopathic RBD (iRBD) eventually develop neurodegenerative disorders, particularly alpha synucleinopathies including Parkinson's disease (PD), dementia with Lewy bodies (DLB), and multiple system atrophy (MSA) [4,5].

Dream enacting behavior in RBD ranges from harmless manifestation such as sleep talking to disruptive behaviors like kicking, punching, falling out of bed [2]. The disruptive behaviors during sleep cause injuries in RBD patients and/or their bed partner [6]. As the current treat- 
ment options, such as clonazepam or melatonin, only reduce the frequency and intensity of symptoms but do not prevent the development of further neurodegenerative disorders [7], disruptive behaviors are the main targets of treatment with medication [8].

So far, however, there has been no detailed investigation of any correlation between the severity of behavioral symptoms and demographic and clinical characteristics in iRBD patients. In this study, we report our findings regarding the demographic and clinical characteristics of iRBD patients with disruptive behavioral symptoms, as well as the risk factors associated with disruptive behavioral symptoms in $\mathrm{RBD}$ patients. We used the REM sleep behavior disorder questionnaire-Hong Kong (RBDQ-HK), a validated measure of clinical RBD symptoms and severity, to evaluate behavioral symptoms in RBD patients [9].

\section{METHODS}

\section{Participants}

Patients diagnosed as having RBD through video-polysomnography (vPSG) at the sleep clinic of Seoul National University Bundang Hospital, Seongnam, Korea were included in the present study. Polysomnography-confirmed RBD patients underwent neurological examination by a neurologist and had neuropsychological tests in order to screen for neurodegenerative disorders. In addition, participants completed three subjective scales: the Pittsburgh Sleep Quality Index (PSQI) for the assessment of sleep quality, the Epworth Sleepiness Scale (ESS) for the assessment of daytime sleepiness, and the Beck Depression Inventory (BDI) for the assessment of depression. Secondary RBD patients with neurodegenerative disorders including PD, MSA, dementia, or narcolepsy were excluded. Individuals who were using antidepressants or clonazepam and individuals who did not complete assessment for neurodegenerative disorders or questionnaires including RBDQ-HK and other subjective scales were also excluded. As data on these individuals with RBD were collected through retrospective review of medical records, written consent was not obtained as it was not required. This study was approved by the Institutional Review Board of the Seoul National University Bundang Hospital (B1408/264-004).

\section{Diagnosis of REM Sleep Behavior Disorder and Video-Polysomnography}

RBD was diagnosed based on the criteria of the International Classification of Sleep Disorders, second edition [1]. A more strict definition of REM sleep without atonia (RSWA) was applied using the American Academy of Sleep Medicine manual [10]. An EmblaTMN7000 device (Embla, Reykjavik, Iceland) was used for polysomnography with simultaneous video recording. EEG electrodes were applied at F4/A1, F3/A2, C4/A1,
$\mathrm{C} 3 / \mathrm{A} 2, \mathrm{O} 1 / \mathrm{A} 2$, and O2/A1 according to the International 1020 System of Electrode Placement, and two electrooculography electrodes were applied on the sides of both eyes. Electromyography electrodes were applied at the submentalis and at both anterior tibialis muscles. Strain gauges were used for recording chest and abdominal respiratory movements, and nasal pressure cannulas were used for airflow. An electrocardiogram was taken, and oxygen saturation was recorded using a pulse oximeter applied to the index finger. We visually scored sleep and sleep stages at every 30-s epoch of the vPSG according to the current criteria with an allowance for RSWA [10].

\section{REM Sleep Behavior Disorder Questionnaire- Hong Kong}

The RBDQ-HK is a 13-item self-administered (by patient and/ or bed partner) questionnaire about both dream-related aspects and the behavioral manifestation of RBD, and it was administered in order to evaluate the RBD symptom severity of participants [9]. Each item assesses symptoms by both lifetime occurrence and recent (1-year) frequency. Items 6 to 12, evaluating behavioral manifestation, are scored with additional weight due to the clinical importance of behavioral symptoms in RBD. The total score is a sum of all items and ranges from 0 to 100 , with a higher score reflecting more frequent symptoms. Participants were divided into two groups according to the presence or absence of disruptive behavioral symptoms based on item 9 (Have you ever fallen from your bed?), item 10 (Have you ever hurt yourself or your bed-partner while you were sleeping?), and item 11 (Have you ever attempted to assault your bed-partner or almost hurt yourself while you were sleeping?) of RBDQHK. Subjects with scores greater than 0 on any of items 9, 10, and 11 were classified as the group with disruptive behavioral symptoms. Scores of item 2 (Did you often have nightmares?), item 3 (Did you have dreams with an emotional and sorrowful content?), item 4 (Did you have dreams with a violent or aggressive content?), and item 5 (Did you have dreams with a frightening and horrifying content?) evaluating dream contents were used in the analysis of risk factors associated with disruptive behavioral symptoms.

\section{Neuropsychological Tests}

The Korean Version of the Consortium to Establish a Registry for Alzheimer's Disease Assessment Packet Neuropsychological Assessment Battery $[11,12]$ as well as additional tests were conducted so as to examine the cognitive performance of participants. Mini-mental status examination was used for global cognitive screening. Trail making test A, digit span forward, and digit span backward were used to assess attention. Memory was evaluated with word list memory, word list recall, word list recognition, and construction recall. Frontal assessment battery and the Stroop color-word interference test were conducted to assess executive functions. The categorical verbal fluency 
test (animal) and Boston naming test were performed for language abilities. Visuospatial abilities were assessed with visuospatial construction, the executive clock drawing task (CLOX2), and the block design test. The Z-scores were calculated from raw scores through demographically adjusting for age, sex, and education.

\section{Statistical Analysis}

Comparisons of demographic variables and Z-scores of neuropsychological tests between the two groups (people with iRBD with or without disruptive behavioral symptoms) were performed using the independent t-test, and the results are reported as means \pm standard deviations. Analysis of covariance (ANCOVA) adjusted by age and sex was used for the subjective scales (PSQI, ESS, BDI) and polysomnographic variables. The results of ANCOVA are reported as adjusted means \pm standard errors. Multiple logistic regression analysis using stepwise backward elimination was performed in order to evaluate risk factors associated with disruptive behavioral symptoms. Demographic and clinical characteristics as well as scores of RBDQ-HK items related to dream contents were the independent variables used in the multiple logistic regression analysis. All significance tests were 2 -sided and the p-value was set at $<0.05$ to achieve statistical significance. Bonferroni correction was applied for neuropsychological tests due to multiple comparisons of cognitive performance (level of significance set at $0.0033=0.05 / 15$ ). SPSS version 22.0 for Windows (IBM Corp., Armonk, NY, USA) was used for data analysis.

\section{RESULTS}

Of the 231 RBD patients diagnosed by vPSG, 188 iRBD patients (122 men and 66 women) remained after the application of exclusion criteria, and these were analyzed (Fig. 1). The mean age of the 188 iRBD patients was $67.3 \pm 7.4$ years (range $44^{-}$ 88 ). They were categorized into two groups according to the presence or absence of disruptive behavioral symptoms, based on items 9, 10, and 11 of RBDQ-HK. The iRBD patients with disruptive behavioral symptoms were younger than those without disruptive behavioral symptoms $(66.8 \pm 7.4$ vs. $70.5 \pm 6.0$, $\mathrm{p}=0.012)$, and showed a higher proportion of men $(69.4 \%$ vs. $39.3 \%, \mathrm{p}=0.004)$. There was no statistically significant difference in BMI, education, PSQI, ESS, or BDI scores between the two groups. The demographic and clinical characteristics of the participants are described in Table 1.

According to polysomnography, iRBD patients with disruptive behavioral symptoms showed lower sleep efficiency (74.6 \pm 0.8 vs. $79.5 \pm 2.1, \mathrm{p}=0.033)$ and longer sleep latency $(29.4 \pm$ 2.5 vs. $13.6 \pm 6.1, \mathrm{p}=0.019$ ) than those without disruptive behavioral symptoms. Apnea-Hypopnea Index (AHI) was lower in general $(9.2 \pm 0.9$ vs. $17.8 \pm 2.2, \mathrm{p}<0.001)$ as well as during

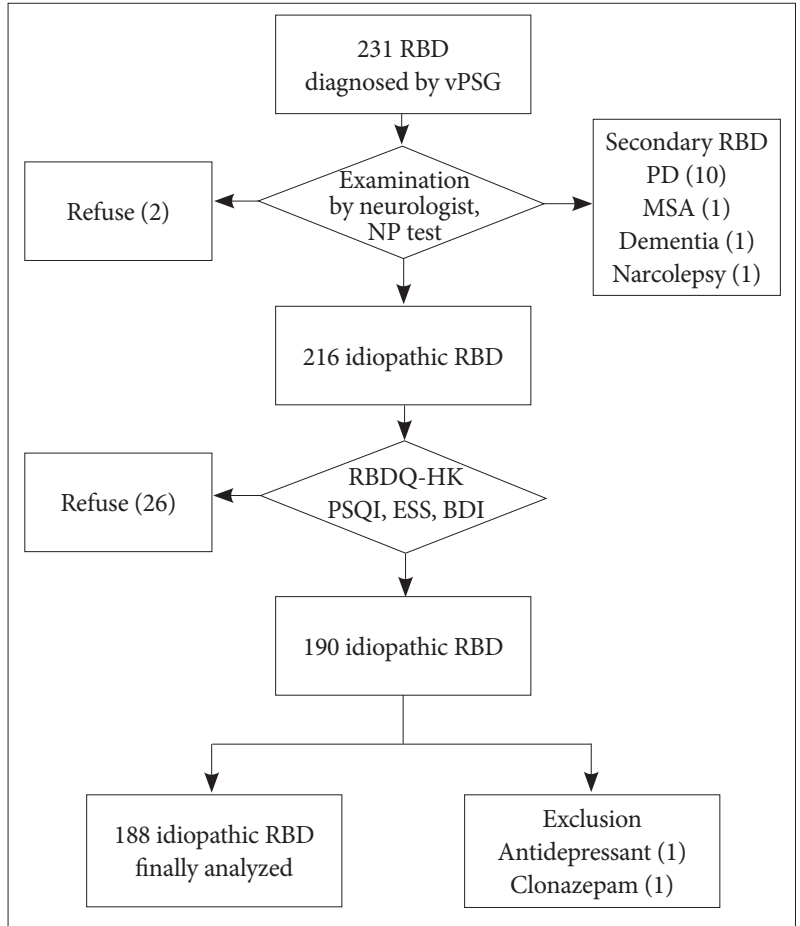

Fig. 1. Flow chart of study subjects. RBD: REM sleep behavior disorder, vPSG: video-polysomnography, NP test: neuropsychological test, PD: Parkinson's disease, MSA: multiple system atrophy, RBDQ-HK: REM sleep behavior disorder questionnaire-Hong Kong, PSQI: Pittsburgh Sleep Quality Index, ESS: Epworth Sleepiness Scale, BDI: Beck Depression Inventory.

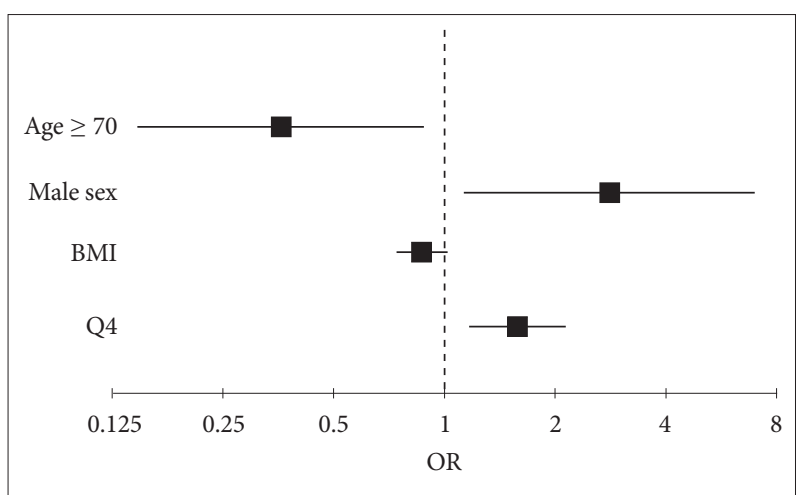

Fig. 2. ORs and $95 \%$ confidence intervals of risk factors of disruptive behavioral symptoms in multiple logistic regression analysis. BMI: body mass index, Q4: score of item 4 (violent or aggressive dreams) of REM sleep behavior disorder questionnaire-Hong Kong, OR: odds ratio.

both REM ( $8.2 \pm 1.0$ vs. $13.8 \pm 2.4, \mathrm{p}=0.033)$ and non-REM sleep $(9.8 \pm 1.0$ vs. $18.2 \pm 2.4, \mathrm{p}=0.002)$ in iRBD patients with disruptive behavioral symptoms (Table 2). Neuropsychological tests showed no statistically significant differences between the two groups in any cognitive domains (Table 3).

In univariate logistic regression analysis, BMI, the score of item 3 (emotional dreams), and the score of item 5 (frightening dreams) of RBDQ-HK, as well as PSQI and BDI showed no statistically significant association with disruptive behavioral 
Table 1. Demographic and clinical characteristics of participants

\begin{tabular}{lccc}
\hline & $\begin{array}{c}\text { Disruptive } \\
\text { behavioral } \\
\text { symptom }(+) \\
(\mathrm{n}=160)\end{array}$ & $\begin{array}{c}\text { Disruptive } \\
\text { behavioral } \\
\text { symptom }(-) \\
(\mathrm{n}=28)\end{array}$ & $\mathrm{p}$ \\
\hline Age (year) & $66.8 \pm 7.4$ & $70.5 \pm 6.0$ & $0.012^{*}$ \\
Sex (male, \%) & $111(69.4)$ & $11(39.3)$ & $0.004^{*}$ \\
BMI (kg/m $\left.)^{2}\right)$ & $24.3 \pm 2.8$ & $25.2 \pm 2.8$ & 0.113 \\
Education (year) & $12.4 \pm 4.6$ & $11.2 \pm 4.3$ & 0.230 \\
PSQI & $6.9 \pm 0.3$ & $6.6 \pm 0.8$ & 0.641 \\
ESS & $7.3 \pm 0.3$ & $5.9 \pm 0.8$ & 0.078 \\
BDI & $7.9 \pm 0.6$ & $8.5 \pm 1.4$ & 0.679 \\
\hline Age, sex, BMI, education
\end{tabular}

Age, sex, BMI, education data are presented as the mean \pm standard deviation. PSQI, ESS, BDI data are presented as the adjusted mean \pm standard error.

${ }^{*} \mathrm{p}<0.05$.

BMI: body mass index, PSQI: Pittsburgh Sleep Quality Index, ESS: Epworth Sleepiness Scale, BDI: Beck Depression Inventory.

Table 2. Polysomnography results

\begin{tabular}{|c|c|c|c|}
\hline & $\begin{array}{c}\text { Disruptive } \\
\text { behavioral } \\
\text { symptom }(+) \\
(\mathrm{n}=160)\end{array}$ & $\begin{array}{c}\text { Disruptive } \\
\text { behavioral } \\
\text { symptom (-) } \\
(\mathrm{n}=28)\end{array}$ & $\mathrm{p}$ \\
\hline Total sleep time (min) & $354.5 \pm 4.38$ & $376.8 \pm 10.8$ & 0.061 \\
\hline Sleep efficiency (\%) & $74.6 \pm 0.8$ & $79.5 \pm 2.1$ & $0.033^{*}$ \\
\hline Sleep latency (min) & $29.4 \pm 2.5$ & $13.6 \pm 6.1$ & $0.019^{*}$ \\
\hline REM latency (min) & $129.3 \pm 6.2$ & $146.1 \pm 15.2$ & 0.313 \\
\hline Wake after sleep onset (min) & $92.0 \pm 3.8$ & $86.6 \pm 9.5$ & 0.597 \\
\hline Stage 1 sleep (\%) & $9.4 \pm 0.4$ & $10.3 \pm 0.9$ & 0.377 \\
\hline Stage 2 sleep (\%) & $44.1 \pm 0.8$ & $49.7 \pm 2.0$ & $0.014^{*}$ \\
\hline Stage 3 sleep (\%) & $10.5 \pm 0.6$ & $7.3 \pm 1.5$ & 0.050 \\
\hline REM sleep (\%) & $17.4 \pm 0.5$ & $15.4 \pm 1.2$ & 0.149 \\
\hline AHI & $9.2 \pm 0.9$ & $17.8 \pm 2.2$ & $<0.001^{*}$ \\
\hline AHI REM & $8.2 \pm 1.0$ & $13.8 \pm 2.4$ & $0.033^{*}$ \\
\hline AHI NREM & $9.8 \pm 1.0$ & $18.2 \pm 2.4$ & $0.002^{*}$ \\
\hline AHI NREM-REM & $1.6 \pm 11.0$ & $4.0 \pm 14.9$ & 0.416 \\
\hline PLMS index & $22.0 \pm 2.7$ & $19.7 \pm 7.0$ & 0.752 \\
\hline
\end{tabular}

Data are presented as the adjusted mean \pm standard error.

${ }^{*} \mathrm{p}<0.05$.

REM: rapid eye movement, AHI: Apnea-Hypopnea Index, AHI REM: AHI during REM sleep, AHI NREM: AHI during non-REM sleep, AHI NREM-REM: AHI NREM-AHI REM.

symptoms in iRBD. After multiple logistic regression analysis, only three variables, age older than or equal to 70 [odds ratio $(\mathrm{OR})=0.36,95 \%$ confidence interval $(\mathrm{CI})=0.15-0.87, \mathrm{p}=0.023)$, male sex $(\mathrm{OR}=2.80,95 \% \mathrm{CI}=1.14-6.91, \mathrm{p}=0.025)$, and score on item 4 (violent or aggressive dreams) of RBDQ-HK (OR = $1.57,95 \% \mathrm{CI}=1.17-2.12, \mathrm{p}=0.003$ ) showed statistically significant association with disruptive behavioral symptoms (Table
Table 3. Neuropsychological test results

\begin{tabular}{|c|c|c|c|}
\hline & $\begin{array}{c}\text { Disruptive } \\
\text { behavioral } \\
\text { symptom }(+) \\
(\mathrm{n}=160)\end{array}$ & $\begin{array}{c}\text { Disruptive } \\
\text { behavioral } \\
\text { symptom (-) } \\
(\mathrm{n}=28)\end{array}$ & $\mathrm{p}$ \\
\hline MMSE & $-0.50 \pm 1.09$ & $-0.29 \pm 1.20$ & 0.353 \\
\hline \multicolumn{4}{|l|}{ Attention } \\
\hline Trail making test $\mathrm{A}$ & $0.87 \pm 0.63$ & $0.74 \pm 0.64$ & 0.316 \\
\hline Digit span forward & $0.33 \pm 0.93$ & $0.05 \pm 1.00$ & 0.148 \\
\hline Digit span backward & $0.36 \pm 1.18$ & $-0.05 \pm 1.04$ & 0.089 \\
\hline \multicolumn{4}{|l|}{ Memory } \\
\hline Word list memory & $0.28 \pm 0.93$ & $0.07 \pm 0.86$ & 0.275 \\
\hline Word list recall & $-0.24 \pm 1.04$ & $-0.65 \pm 0.91$ & 0.052 \\
\hline Word list recognition & $-0.14 \pm 0.99$ & $-0.06 \pm 0.84$ & 0.685 \\
\hline Construction recall & $0.06 \pm 1.01$ & $-0.10 \pm 0.98$ & 0.464 \\
\hline \multicolumn{4}{|l|}{ Executive functions } \\
\hline Frontal assessment battery & $0.25 \pm 0.75$ & $-0.07 \pm 0.90$ & 0.042 \\
\hline $\begin{array}{c}\text { Stroop color-word } \\
\text { interference test }\end{array}$ & $0.15 \pm 1.04$ & $-0.15 \pm 1.18$ & 0.169 \\
\hline \multicolumn{4}{|l|}{ Language abilities } \\
\hline $\begin{array}{l}\text { Categorical verbal } \\
\text { fluency test }\end{array}$ & $0.37 \pm 1.15$ & $0.33 \pm 1.05$ & 0.860 \\
\hline Boston naming test & $0.66 \pm 0.83$ & $0.71 \pm 0.80$ & 0.771 \\
\hline \multicolumn{4}{|l|}{ Visuospatial abilities } \\
\hline Visuospatial construction & $0.08 \pm 0.81$ & $0.17 \pm 0.88$ & 0.597 \\
\hline $\begin{array}{l}\text { Executive clock } \\
\text { drawing task }\end{array}$ & $0.29 \pm 0.49$ & $0.19 \pm 0.77$ & 0.486 \\
\hline Block design test & $0.50 \pm 1.05$ & $0.43 \pm 1.10$ & 0.765 \\
\hline
\end{tabular}

$\mathrm{Z}$-scores are presented as the mean \pm standard deviation. MMSE: mini-mental status examination.

4). In addition, a score of item 4 of RBDQ-HK was higher in men than in women $(2.6 \pm 1.3$ vs. $2.1 \pm 1.8, \mathrm{p}=0.041)$, while the other dream-related items, including items 2 (frequent nightmares), 3, and 5, did not showed significant sex differences.

\section{DISCUSSION}

The present study aimed to investigate the demographic and clinical characteristics of iRBD patients with disruptive behavioral symptoms. We found a younger age, male preponderance, lower sleep efficiency, longer sleep latency, lower AHI in the iRBD group with disruptive behavioral symptoms than the iRBD group without them, and we also found no statistically significant difference in cognitive performance. Multiple logistic regression analysis showed that an age younger than 70 , male sex, and a high frequency of violent or aggressive dream content increase the risk of disruptive behavioral symptoms in $\mathrm{iRBD}$.

Although disruptive behavioral symptoms are the main treat- 
Table 4. Adjusted ORs and $95 \%$ Cls for disruptive behavioral symptoms

\begin{tabular}{|c|c|c|c|c|}
\hline \multirow{2}{*}{ Variables } & \multicolumn{2}{|c|}{ Univariate logistic regression } & \multicolumn{2}{|c|}{ Multiple logistic regression } \\
\hline & OR $(95 \%$ CI $)$ & $\mathrm{p}$ & OR $(95 \%$ CI $)$ & $\mathrm{p}$ \\
\hline Age $\geq 70$ & $0.35(0.15-0.80)$ & $0.012^{*}$ & $0.36(0.15-0.87)$ & $0.023^{*}$ \\
\hline Male sex & $3.50(1.53-8.03)$ & $0.003^{*}$ & $2.80(1.14-6.91)$ & $0.025^{*}$ \\
\hline $\operatorname{BMI}\left(\mathrm{kg} / \mathrm{m}^{2}\right)$ & $0.89(0.77-1.03)$ & 0.115 & $0.87(0.75-1.01)$ & 0.071 \\
\hline Q2 (frequent nightmares) & $1.32(1.03-1.69)$ & $0.031^{*}$ & & \\
\hline Q3 (emotional dreams) & $1.32(0.91-1.92)$ & 0.147 & & \\
\hline Q4 (violent or aggressive dreams) & $1.67(1.26-2.22)$ & $<0.001^{*}$ & $1.57(1.17-2.12)$ & $0.003^{*}$ \\
\hline Q5 (frightening dreams) & $1.04(0.81-1.35)$ & 0.749 & & \\
\hline PSQI & $0.99(0.90-1.10)$ & 0.882 & & \\
\hline BDI & $0.98(0.93-1.03)$ & 0.356 & & \\
\hline
\end{tabular}

${ }^{*} \mathrm{p}<0.05$.

OR: odds ratio, CI: confidence interval, BMI: body mass index, Q2: score of item 2 (frequent nightmares) of REM sleep behavior disorder questionnaire-Hong Kong (RBDQ-HK), Q3: score of item 3 (emotional dreams) of RBDQ-HK, Q4: score of item 4 (violent or aggressive dreams) of RBDQ-HK, Q5: score of item 5 (frightening dreams) of RBDQ-HK, PSQI: Pittsburgh Sleep Quality Index, BDI: Beck Depression Inventory.

ment target of RBD, until now, far too little attention has been paid to the correlations of these symptoms with other factors. One previous study on the factors associated with injury in RBD reported a higher probability and severity of injury in $\mathrm{iRBD}$ patients than in secondary RBD patients, and demonstrated that recall of dreams, not frequency of dream enacting behavior, was a risk factor of injury. However, this is the first study to evaluate the various factors associated with disruptive behavior itself in iRBD [13].

The present study shows that male sex and frequent violent or aggressive dream contents are risk factors for disruptive behavioral symptoms in iRBD. RBD itself shows a male preponderance, however, the cause of this is unknown [14]. One hypothesis is that women with RBD tend to exhibit fewer aggressive and violent behaviors [15], leading to fewer clinic visits and under-recognition of the disease. One study reported that subclinically-increased submental tonic EMG activity in REM sleep, which indicates the mildest case of RBD, had equal prevalence in both men and women with PD, but that RBD with clinically evident severity was more frequent in men [16]. This hypothesis can also be applied to the male preponderance in iRBD patients with disruptive behavioral symptoms found in our study. The difference between RBD-related dream content in men and women can also partially explain this sex disparity. Previous research found that men with PD had more aggressive dream content than women with $\mathrm{PD}$, but this sex difference did not reach a statistically significant level in RBD patients [17]. However, in the current study, men with iRBD scored higher on the item measuring frequency of violent or aggressive dream contents than women with $\mathrm{RBD}$, and this higher frequency was also found as a significant risk factor for disruptive behavioral symptoms. Even after adjusting for the correlation between the male sex and the frequency of violent or aggressive dream content, both were still independent risk factors for disruptive behavioral symptoms in iRBD.

One of the important pathogeneses of obstructive sleep apnea (OSA) is a decreased tone of muscle related to the collapse of a pharyngeal airway, such as the genioglossal muscle [18]. The hypothesis that maintenance of muscle tone during REM sleep in RBD might have a protective effect against OSA has been repeatedly suggested [19]. However, some studies have shown inconsistent results, reporting no protective effect [20]. Moreover, whether increased EMG activity in RBD has a protective effect against cardiovascular and metabolic complication of OSA is also uncertain [21]. Lower AHI was found in the iRBD group with disruptive behavior than the group without disruptive behavior in the present study, and this can be explained by the hypothesis mentioned above.

An age below 70 might be a risk factor for disruptive behavioral symptoms because severe behavioral manifestation can shorten the duration between disease onset and its diagnosis. The bed partner's report is crucial in the detection of RBD, as many patients miss their own abnormal behaviors during sleep [22]. Thus, dream enacting behaviors severe enough to harm oneself or his/her bed partner might lead to the earlier detection of RBD. Furthermore, an age-related decrease in muscle strength may be another reason for less severe behavioral manifestation in older patients. On the other hand, it can be hypothesized that iRBD with more severe behavioral manifestation might have an earlier onset of disease, but that a tendency of less violent behaviors in early-onset RBD before age 50 is partly in contrast with this possibility [23]. Due to the lack of information on the duration of illness prior to diagnosis, it is hard to infer the exact reason for age below 70 being a risk factor for disruptive behavioral symptoms.

Several reports have shown cognitive dysfunction in iRBD, 
mainly in the domains of visuospatial abilities, attention, and executive function [24,25], and cognitive dysfunction tends to progress over time [26]. There was no statistically significant difference in neuropsychological test results between $\mathrm{RBD}$ with disruptive behavioral symptoms and $\mathrm{iRBD}$ without disruptive behavioral symptoms found in the present study. Although REM sleep regulation is related to nuclei in the brainstem, the neurodegenerative process in $\mathrm{RBD}$ involves other areas including nigrostriatal system and cortical structures [27]. However, disruptive behavior is thought to be related to the dorsal midbrain and pons rather than cortical structures, thus it is plausible that no difference in cognitive performance according to disruptive behavioral symptoms was found in the current study. One study also reported that increased tonic chin EMG activity during REM sleep at the initial diagnosis of iRBD predicted a higher conversion rate to PD but was not correlated with dementia [28]. There has been a previous study reporting the risk factors of conversion from iRBD to neurodegenerative synucleinopathy. Several such risk factors were suggested, including advanced age, olfactory loss, abnormal color vision, subtle motor dysfunction, and nonuse of antidepressants, as predictors of $\mathrm{PD}, \mathrm{DLB}$, and MSA in iRBD patients [29]. However, no previous study has investigated the correlation between the severity of behavioral symptoms in iRBD and the conversion to neurodegenerative disease. In order to develop a full picture of iRBD as a prodromal stage of synucleinopathy, additional studies will be needed that investigate the further implications of disruptive behavioral symptoms in iRBD.

The current study has several limitations: First, although there has been research on the validity and reliability of RBDQ-HK as a measure of RBD symptoms and severity [9], the present study still lacks an objective measure of disruptive behaviors. The analysis of chin and leg EMG activities might provide more reliable and quantitative information on disruptive behavioral symptoms. Secondly, as mentioned above, the duration of illness prior to diagnosis was not considered. This hinders further inference on age and its association with disruptive behavioral symptoms. However, intrinsic difficulties might lie in determining the exact onset of RBD symptom, as the mildest form of RBD is hard to detect without polysomnography. The small sample size of patients with neurodegenerative disorders is another limitation, as we were not able to analyze this population, and as a result they were excluded. Further study on the correlation between disruptive behavioral symptoms and the neurodegenerative process is needed. In addition, as the study subjects were from a sleep clinic, the number of RBD patients without disruptive behaviors was small compared to that of RBD patients with disruptive behaviors.

In conclusion, this is the first study to explore the factors associated with disruptive behavioral symptoms in iRBD. Considering the growing importance of $\mathrm{iRBD}$ as a prodromal stage of neurodegenerative disorders, further studies on the demo- graphic and clinical characteristics of iRBD patients with disruptive behaviors are needed in order to better understand the pathophysiology of the disease and to establish a robust treatment and management guideline.

\section{Conflicts of Interest}

The authors have no financial conflicts of interest.

\section{Authors' Contribution}

Conceptualization: Ryu SK, Kim MJ. Data curation: Choi HY, Lee HJ. Formal analysis: Ryu SK. Writing_original draft: Ryu SK. Writing-review \& editing: Ryu SK, Yoon IY. Supervision: Yoon IY.

\section{REFERENCES}

1. AASM. International Classification of Sleep Disorders: Diagnostic and Coding Manual. Westchester: American Academy of Sleep Medicine 2005.

2. Rodriguez CL, Jaimchariyatam N, Budur K. Rapid eye movement sleep behavior disorder: a review of the literature and update on current concepts. Chest 2017;152:650-62.

3. Kang SH, Yoon IY, Lee SD, Han JW, Kim TH, Kim KW. REM sleep behavior disorder in the Korean elderly population: prevalence and clinical characteristics. Sleep 2013;36:1147-52.

4. Howell MJ, Schenck CH. Rapid eye movement sleep behavior disorder and neurodegenerative disease. JAMA Neurol 2015;72:707-12.

5. Savica R, Bradley BF, Mielke MM. When do a-synucleinopathies start? An epidemiological timeline: a review. JAMA Neurol 2018;75:503-9.

6. Comella CL, Nardine TM, Diederich NJ, Stebbins GT. Sleep-related violence, injury, and REM sleep behavior disorder in Parkinson's disease. Neurology 1998;51:526-9.

7. Peall KJ, Robertson NR. Idiopathic rapid eye movement sleep behaviour disorder: a potential gateway to the development of disease-modifying treatments in neurodegenerative disorders. J Neurol 2016;263:1678-80.

8. Chiu HF, Wing YK, Lam LC, Li SW, Lum CM, Leung T, et al. Sleep-related injury in the elderly--an epidemiological study in Hong Kong. Sleep 2000;23:513-7.

9. Li SX, Wing YK, Lam SP, Zhang J, Yu MW, Ho CK, et al. Validation of a new REM sleep behavior disorder questionnaire (RBDQ-HK). Sleep Med 2010;11:43-8.

10. Iber C, Ancoli-Israel S, Chesson AL, Quan SF. The AASM Manual for the Scoring of Sleep and Associated Events. Rules, Terminology and Technical Specifications. 1st ed. Westchester: American Academy of Sleep Medicine 2007.

11. Lee JH, Lee KU, Lee DY, Kim KW, Jhoo JH, Kim JH, et al. Development of the Korean version of the consortium to establish a registry for Alzheimer's Disease Assessment Packet (CERAD-K): clinical and neuropsychological assessment batteries. J Gerontol B Psychol Sci Soc Sci 2002;57:47-53.

12. Lee DY, Lee KU, Lee JH, Kim KW, Jhoo JH, Kim SY, et al. A normative study of the CERAD neuropsychological assessment battery in the Korean elderly. J Int Neuropsychol Soc 2004;10:72-81.

13. McCarter SJ, St Louis EK, Boswell CL, Dueffert LG, Slocumb N, Boeve $\mathrm{BF}$, et al. Factors associated with injury in REM sleep behavior disorder. Sleep Med 2014;15:1332-8.

14. Iranzo A, Santamaria J, Tolosa E. The clinical and pathophysiological relevance of REM sleep behavior disorder in neurodegenerative diseases. Sleep Med Rev 2009;13:385-401.

15. Bodkin CL, Schenck CH. Rapid eye movement sleep behavior disorder in women: relevance to general and specialty medical practice. $J$ Womens Health (Larchmt) 2009;18:1955-63.

16. Gagnon JF, Bédard MA, Fantini ML, Petit D, Panisset M, Rompré S, et al. REM sleep behavior disorder and REM sleep without atonia in Parkinson's disease. Neurology 2002;59:585-9. 
17. Borek LL, Kohn R, Friedman JH. Phenomenology of dreams in Parkinson's disease. Mov Disord 2007;22:198-202.

18. Dempsey JA, Veasey SC, Morgan BJ, O’Donnell CP. Pathophysiology of sleep apnea. Physiol Rev 2010;90:47-112.

19. Bugalho P, Mendonça M, Barbosa R, Salavisa M. The influence of sleep disordered breathing in REM sleep behavior disorder. Sleep Med 2017;37:210-5.

20. Cochen De Cock V, Abouda M, Leu S, Oudiette D, Roze E, Vidailhet $\mathrm{M}$, et al. Is obstructive sleep apnea a problem in Parkinson's disease? Sleep Med 2010;11:247-52.

21. Zhou J, Zhang J, Lam SP, Mok V, Chan A, Li SX, et al. Mortality and its risk factors in patients with rapid eye movement sleep behavior disorder. Sleep 2016;39:1543-50.

22. Iranzo A. The importance of sleep medicine consultation for diagnosis of REM sleep behavior disorder in most patients with Parkinson's disease. Sleep Med 2002;3:537-8.

23. Ju YE. Rapid eye movement sleep behavior disorder in adults younger than 50 years of age. Sleep Med 2013;14:768-74.
24. Manni R, Sinforiani E, Pacchetti C, Zucchella C, Cremascoli R, Terzaghi M. Cognitive dysfunction and REM sleep behavior disorder: key findings in the literature and preliminary longitudinal findings. Int $J$ Psychophysiol 2013;89:213-7.

25. Gagnon JF, Bertrand JA, Génier Marchand D. Cognition in rapid eye movement sleep behavior disorder. Front Neurol 2012;3:82.

26. Youn S, Kim T, Yoon IY, Jeong J, Kim HY, Han JW, et al. Progression of cognitive impairments in idiopathic REM sleep behaviour disorder. $J$ Neurol Neurosurg Psychiatry 2016;87:890-6.

27. Boeve BF, Silber MH, Saper CB, Ferman TJ, Dickson DW, Parisi JE, et al. Pathophysiology of REM sleep behaviour disorder and relevance to neurodegenerative disease. Brain 2007;130:2770-88.

28. Postuma RB, Gagnon JF, Rompré S, Montplaisir JY. Severity of REM atonia loss in idiopathic REM sleep behavior disorder predicts Parkinson disease. Neurology 2010;74:239-44.

29. Postuma RB, Gagnon JF, Bertrand JA, Génier Marchand D, Montplaisir JY. Parkinson risk in idiopathic REM sleep behavior disorder: preparing for neuroprotective trials. Neurology 2015;84:1104-13. 